\title{
TOWARDS DEEP LEARNING FOR ARCHITECTURE: A MONUMENT RECOGNITION MOBILE APP
}

\author{
V. Palma ${ }^{1}$ \\ ${ }^{1}$ FULL_Future Urban Legacy Lab, Politecnico di Torino, Torino, Italy, valerio.palma@polito.it
}

\begin{abstract}
KEY WORDS: Artificial Intelligence, Machine Learning, Deep Learning, Convolutional Neural Networks, Architectural Heritage,
\end{abstract} Mobile Apps, Information Modeling

\begin{abstract}
:
In recent years, the diffusion of large image datasets and an unprecedented computational power have boosted the development of a class of artificial intelligence (AI) algorithms referred to as deep learning (DL). Among DL methods, convolutional neural networks $(\mathrm{CNNs})$ have proven particularly effective in computer vision, finding applications in many disciplines. This paper introduces a project aimed at studying CNN techniques in the field of architectural heritage, a still to be developed research stream. The first steps and results in the development of a mobile app to recognize monuments are discussed. While AI is just beginning to interact with the built environment through mobile devices, heritage technologies have long been producing and exploring digital models and spatial archives. The interaction between DL algorithms and state-of-the-art information modeling is addressed, as an opportunity to both exploit heritage collections and optimize new object recognition techniques.
\end{abstract}

\section{INTRODUCTION}

The city is now producing a brand-new information on itself, in terms of quality and kind, in the form of data that can be stored, organized, analyzed. Current technical needs are format standardization, information gathering, management and selection, data processing and visualization... This is leading unprecedented developments of the tools.

New networks of relationships among documents can be defined and rapidly redefined, according to continuously updating needs and contents. In this complex information topology, extended by the digital archives, the physical form of architecture maintains a key role, on which even the most up-to-date elaborations can be founded. A specific approach to automated architecture recognition could change the way in which data on the urban environment are collected, processed and analyzed, and could provide new ways to access a spatialized information network.

The project under the working name SHAZARCH intends to enhance the connection between the physical and digital space starting from architectural heritage sites. Since many works on the digitalization of cultural sites and related archives have already been started, the heritage field is well suited for the development of an informative tool based on object recognition. In order to deal with these issues, we are studying the technological domain that is commonly called 'artificial intelligence' (AI). More precisely, convolutional neural network (CNN) techniques are addressed. Thanks to their diffusion even on mobile devices, these digital tools can increasingly interact with the urban environment.

As a first case study, we applied a CNN-based software in a mobile app for the Archaeological Central Area in Rome which includes the Imperial Fora.

Starting from this first experiment, the research tries to extend its questions to more general problems related to AI. This technology allows to filter and order large amounts of data: can it be an opportunity to produce new kinds of maps? What are the limitations and the threats in automatic architecture recognition processes?

The project involves cooperation between different research teams and institutions. A team from the Università di Roma Tre has been carrying on the technical operations related to the machine learning model. The ReLOAD lab of the Università di Padova maintains the web platform Cult (CULT project, 2018), which has been used to store and access data and documents. The FULL Interdepartmental Center of the Politecnico di Torino is currently developing software components and field applications of the results. A commercial partner has made possible the production of a first mobile app for the digital market exploiting SHAZARCH technologies.

\section{RELATED WORKS}

\subsection{Deep learning and monument recognition}

AI is now experiencing a very fast development. Deep learning (DL) refers to a class of algorithms in the vast realm of machine learning (ML) that is suitable for a range of challenging tasks such as recognizing objects. These models are capable "to learn complicated concepts by building them out of simpler ones" (Goodfellow et al., 2016). DL founding methods date back to 1940s and key advancements came between the 1980s and the $1990 \mathrm{~s}$, though the computational effort required to train these models prevented them to outclass other ML algorithms. DL is now emerging and gaining industrial interest thanks also to the unprecedented computing power available.

Among DL models, convolutional neural networks (CNNs) feature specialized architectures for computer vision. CNNs are currently employed for image recognition in medicine, biology and many other research fields (Hosny et al., 2018; Webb, 2018). Nevertheless, only a few recent studies are employing these technologies for architecture-related research.

The interest in monument and landmark recognition comes before the latest advancements in DL. Chen et al. (2009) and Amato et al. (2015) present reviews of such applications of image recognition techniques. Since 2012, CNNs started outperforming previously used algorithms in detecting and classifying objects from images (Amato et al., 2016). The introduction of CNN in heritage studies is proposed by Amato et al. (2016), whose target objects are ancient inscriptions and a set of 12 monuments in Pisa (Italy), and more recently by Gada et al. (2018) with an application to a set of 12 famous Indian monuments.

Advancements emerge from other research streams exploring the built environment. Due to applications related to self-driving cars, computer vision is extensively applied to street-level imagery. In this field, Cordts et al. (2016) show that CNNs can be used to perform image segmentation, which is the classification of image portions into sets of categories. In this as in other cases, the availability of vast and public datasets has been a boost for the development of DL models, allowing for training, testing and benchmarking (Goodfellow et al., 2016). 
It is worth noting that on the dataset repository available on Kaggle - a platform hosting data prediction competitions, gathering a large community of data scientists - to this day only a slight interest is shown for architecture- and heritage-oriented computer vision (Kaggle Inc., 2018).

Much attention has still to be paid on finding an optimal AI model to classify architectural objects and features.

\subsection{Digital archives}

The development of digital survey and graphic processing tools multiplies the outputs and contributes to increasingly detailed representations. Due to the ever-growing collected information, many studies on cultural heritage are already paying high attention to the advantages of information and communication technologies (ICTs) for data accessibility.

A particular interest in employing AI techniques in heritage fields emerges from a flourishing research on spatial information and digital archives.

Facing a structural heterogeneity of data and documents, cultural heritage collections rely on metadata interoperability and integration. Many studies (Oldman et al., 2014; Eide et al., 2008; Kakali et al., 2007) confirmed the role of metadata schemas such as the infrastructures of the Semantic Web (Berners-Lee et al., 2001) and the CIDOC CRM ontology (Doerr, 2003).

As Saygi and Remondino (2013) highlight, architectural heritage expresses a specific need for the management of the spatial characteristics of the studied assets. This led to successful applications of information modeling tools, in which a spatial representation can be connected to sets of attributes stored in a database. Among these tools, geographic information systems (GIS) have long been a common approach for storing and managing spatial information related to cultural sites and artworks, and though specialized in 2D geographic data, they can include temporal information and 2.5D-3D representations (Soler et al., 2017; Cerutti et al., 2015; Apollonio et al., 2012).

More recently, building information modelling (BIM) emerged as a tool for more complex operations on the $3 \mathrm{D}$ model. The use of this technology, derived from the engineering and construction industry, introduced the concept of an historical BIM, or HBIM (Murphy et al., 2009). Volk et al. (2014) present an extensive review of BIM applications to existing buildings.

The studies by Saygi and Remondino (2013) and Saygi et al. (2013) directly compare GIS and BIM as approaches for heritage applications, while, in a broader disciplinary perspective, other studies are coping with the integration of the two systems (Liu et al. 2017, Song et al., 2017; Hor et al., 2016).

Another task pursued through the digitalization of heritage archives is enabling multimedia applications, for visualization or analysis purposes (Auer et al., 2014; Scianna et al., 2013).

Among current works presenting advancements in this direction, the Arches project and the INCEPTION project can be cited (Arches project, 2018; INCEPTION project, 2018; Maietti et al., 2018; Myers et al., 2016). Both of these are dealing with standard compliant collections, with the aim to make an interoperable information available through multiple services like websites, digital maps, apps...

Similar issues are faced by the team from the Università di Padova which is working at the present project. The Cult platform (Cult project, 2018) is a bundle of software tools to store data from architectural heritage research projects and to share them with websites, tourist apps and BIM and GIS interfaces.

\section{APP OVERVIEW}

The digitalization of cultural sites and related archives offers a structured and ready-to-use source of information that can be retrieved through the flexible features of DL object recognition algorithms.
The present research developed a mobile app that allows the user to access data related to a building or work of art just by pointing the camera at the object. The result is capable of connecting the real city to a set of documents such as images, texts, 3D models and others. It builds on original developments of $\mathrm{CNN}$ techniques aimed at recognizing architectural objects.

The app is based on two main blocks of software (Figure 1). A first one is a database with the following features: it is online; it makes it possible to upload different types of document and the related information or metadata; it is geographic-enabled - that is, it can be analyzed and edited through GIS tools. A second piece of software is the ML part. It presents the following features: it is stored on the device; it requires a small amount of disk space; it downloads information on monuments from the online database; it localizes the user.

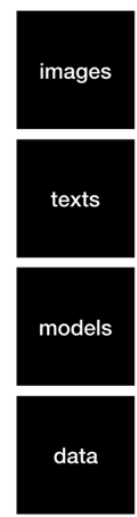

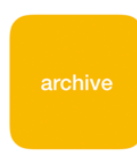

\section{- online}

- multimedia

- geographic
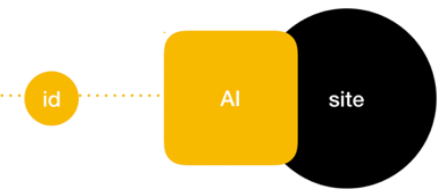

- on device

- geolocated
Figure 1. Scheme of the two main components of the app: the digital archive, storing data and documents, and the artificial intelligence engine, interacting with the site through the app.

The app becomes the access point to an unexploited network of digital information, not through a catalogue or a predefined route on a map, but just by looking at the urban context. A vast multimedia information can be linked to the elements of a city, answering questions on how to make the newly available information easily and sustainably reachable.

The light weight 'brain' exploits a distinctive characteristic of CNN models which is the small disk storage needed (Howard et al., 2017). In fact, DL models do not derive their 'knowledge' from hard-coded information (i.e. no pictures are stored), but rather learn a method to recognize things. In the proposed application, this solution is particularly suitable for tourists, who may not have an internet connection while abroad.

An interesting challenge is posed by choosing the Archaeological Central Area in Rome as a test field. The problem of providing appropriate informative services on site is addressed. This task cannot be easily accomplished by means of informative panels, audio tours or QR codes, for the needed infrastructure may not be compatible with the precious site and the number of visitors. The proposed app needs just a smartphone to make data available a large amount of data. Thus, this information has to be precise and complete, and it should be suitable for many kinds of users. Another challenge relates to the application of CNNs to the monuments inside a restricted perimeter - compared with many previous attempts found in literature. Objects in the same cultural site may indeed be very similar, or may appear together in the same view, making it difficult to distinguish an element from another. However, these conditions stress the practical utility of AI more than the recognition of landmarks which are very far from each other. Hence, our work suggests the possibility to apply the most recent computer vision techniques to get a more detailed information than before. 


\section{APP DEVELOPMENT}

We produced a complete and market-ready Apple iOS app. The app was developed using the Xcode integrated development environment (IDE) and the Swift programming language (Apple Inc., 2018). Xcode includes the Core ML framework to manage ML models. Models must be converted to the Core ML model format using tools provided by Apple. Many models and thirdparty frameworks are supported for conversion, including the employed library Keras.

More limited experiments were conducted using Android prototype apps. These were developed using the Android Studio IDE (Google Inc., JetBrains s.r.o., 2018) (Figure 2).

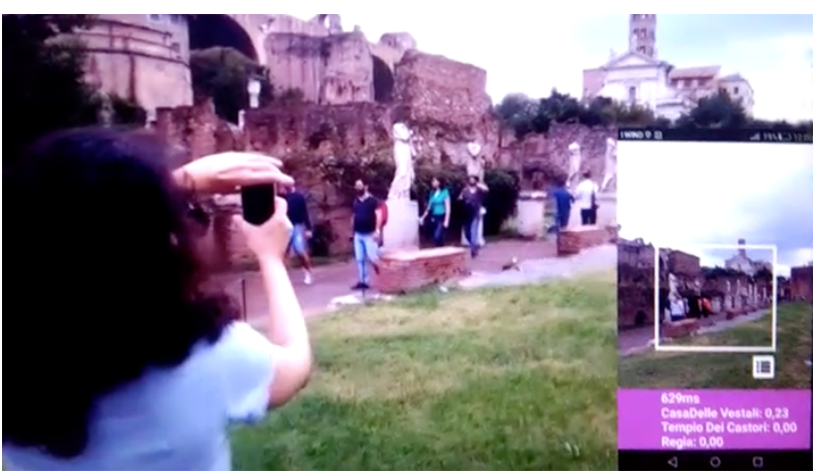

Figure 2. Field tests of an Android prototype of the recognition app (courtesy of Désirée Adiutori).

\subsection{Training dataset collection}

AI technology is capable of assigning labels to input images, on the basis of a training process during which the AI learns from a set of manually labeled images.

The model has been trained to recognize 46 monuments of different size and conservation status. Examples are the Umbilicus Urbis Romae - a cylindric structure less than 5 meters large - , the Domus Augustana - a vast palace on the Palatine Hill —, the Temple of Antoninus and Faustina showing a portico with six columns, now part of the church of San Lorenzo in Miranda - or the Aedes Concordiae - a poorly preserved temple of which not much more than the podium can be seen.

Our dataset is composed of a first set of about 50 to 100 pictures for each monument, which were expressly taken for this project (Figure 3). We chose the poses in order to get an exhaustive overview of the monument and specific attention was given to the most favorable conditions for a visitor, taking details and panoramic views into account.

In order to produce a larger dataset, the first set of pictures was extended creating altered versions of the originals. In fact, data augmentation techniques have proven particularly effective for DL object recognition (Goodfellow et al., 2016). Operations such as translation, rotation, scaling, noise injection and color alterations were performed. Our augmented dataset consisted of 500 images per monument. Finally, all the pictures were cropped and down-sampled to a size of $224 \times 224$ pixels, for the training system requires fixed input dimensions.

In order to be used in the training phases, images were organized into labeled folders, each one corresponding to one monument.

\subsection{Training and testing}

The employed CNN algorithm is based on the MobileNet model (Howard et al., 2017) and was coded in Python language using the open source libraries TensorFlow (Google Inc. and Tang, 2018) and Keras (Chollet et al., 2018). Training was performed on a NVIDIA Tesla GPU cluster. Though the training operations were time expensive, one of the advantages of this system is that a model can be re-trained on new images starting from the previous status.

A first series of tests on the model effectiveness has been conducted, resulting in corrections and useful consideration for the future of the project.

The overall accuracy of the trained models, computed on the basis of a trial subset of images, was estimated to be over $95 \%$. Field tests in different lighting conditions showed heterogeneous results across the different monuments and less reliable recognitions in some specific cases.

Tests also revealed that the majority of non-matching results was mistaken for the Domus Augustana. We identified the extension of the imperial palace and the diversity of its parts as confounding input for the AI. We obtained better results by dividing the training images in four groups, each one relating to a part of the building. This suggests that further work and evaluations can be done on labeling subsets of the training images also in the case of smaller objects.

More works on the technical aspects and the benchmarking of the used ML techniques will follow.

The storage used by the resulting Core ML model is $13.1 \mathrm{MB}$, achieving the goal of a light weight recognition engine that does not need a network connection to provide a basic information on the selected monuments. We expect this same model to be capable of recognizing about 1000 monuments without sensible loss of accuracy.
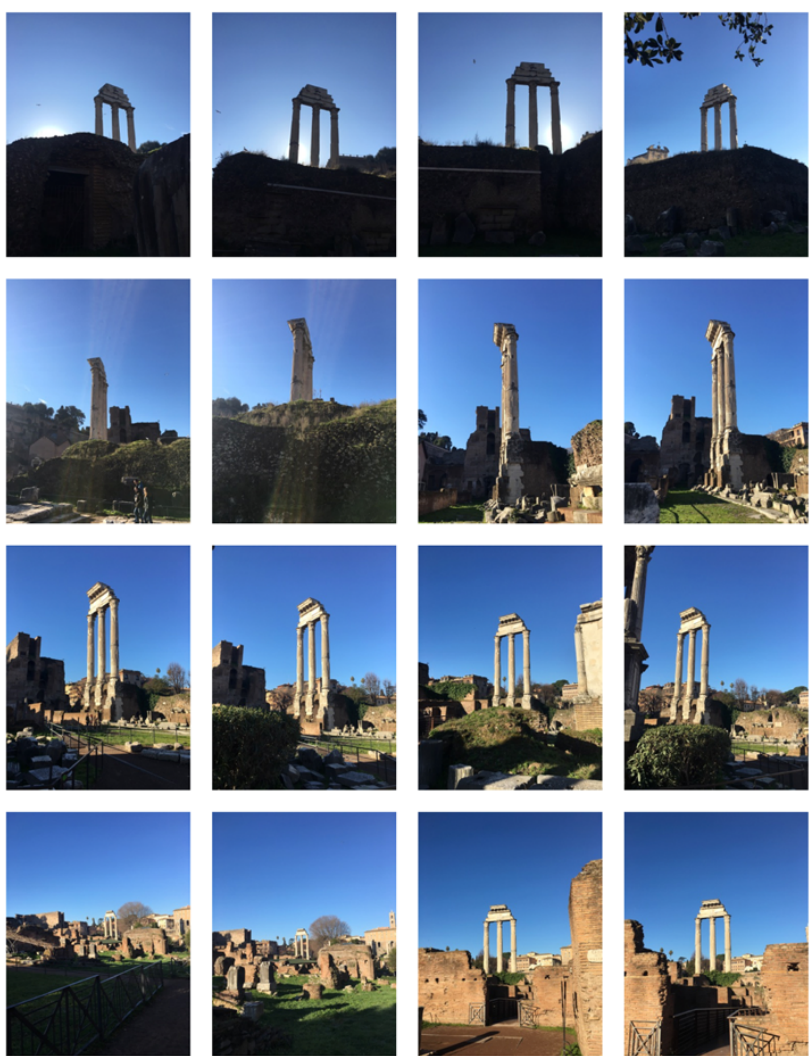

Figure 3. Sample from the original (not augmented) set of training images for the Temple of Castor and Pollux. Panoramic

views and pictures showing not optimal lighting conditions were maintained to simulate user's conditions. 

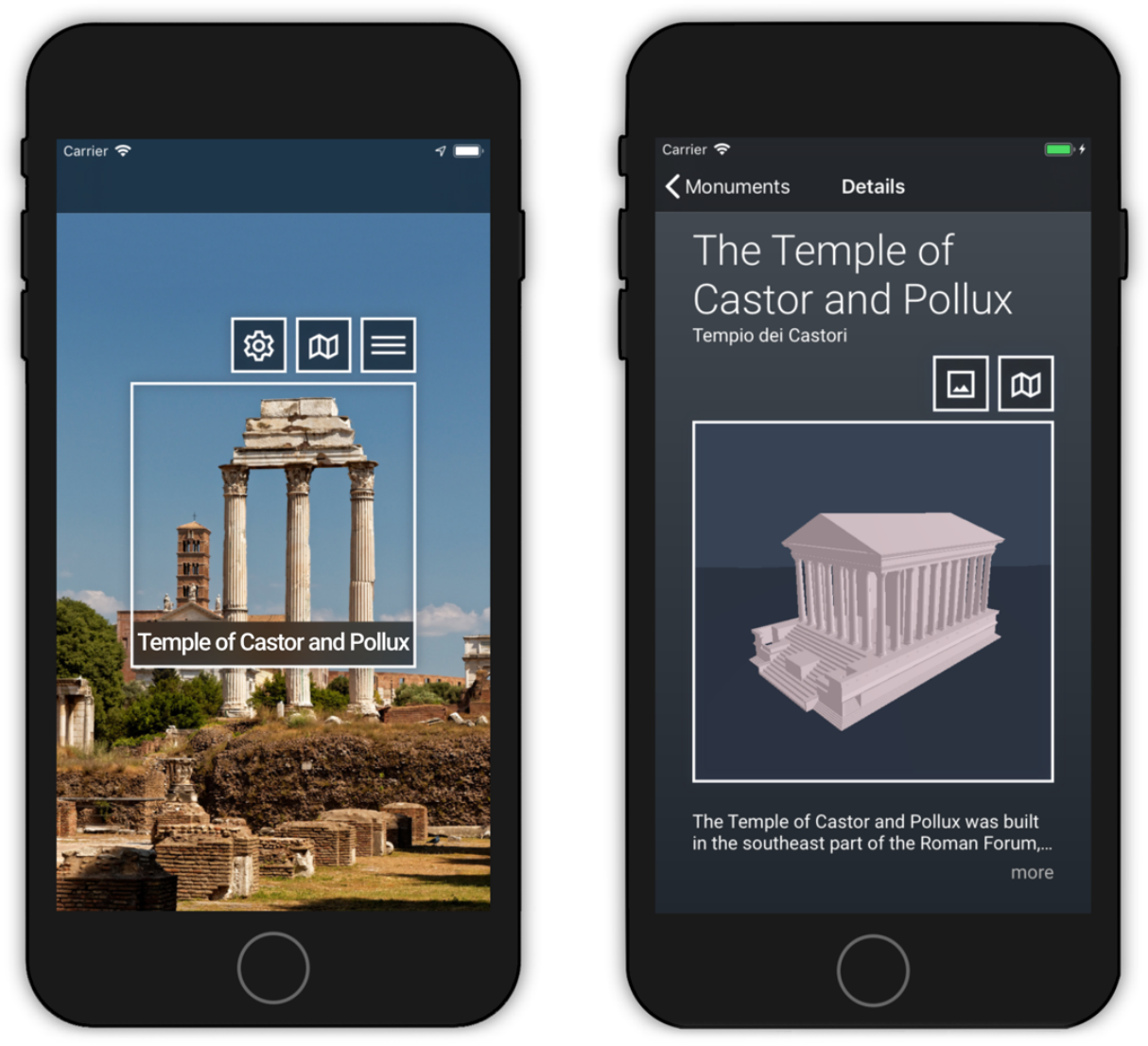

Figure 4. Screenshots of the App. The main page shows the camera monitor, a square to frame monuments and the prediction label. The monument page hosts a description, the interactive $3 \mathrm{D}$ reconstruction and buttons to show images and maps.

\subsection{Database connection and user interface}

We worked on a user-friendly app focused on the speed of use. The app consists of two main screens or pages (Figure 4).

1. The main screen features a minimal design: it shows images from the camera stream on background and draws a square to frame the target monument. ML recognition runs continuously, but the app displays results only when the computed prediction accuracy exceeds a pre-determined threshold (values greater than $80 \%$ were regarded as reliable answers). When a monument name is shown, touching into the squared frame redirects to the monument details.

2. The monument details page layouts the information set previously downloaded from the online database. For the Imperial Forum application, we integrated 3D explorable reconstruction models.

The app interacts with the Cult database, in which data for the monuments covered by the ML training dataset were inserted (Figure 5). Cult hosts web-based services to upload texts, images and models and to associate metadata to each document. It also allows to explore the contents through a website or using GIS applications. The service is entirely based on free and open source software including PostgreSQL for database management, PostGIS as geographic extension and the Django framework for web development (Bortot et al., 2017).

Cult metadata are organized on the basis of the Dublin Core Metadata Initiative scheme (DCMI, 2018). The 15 entries of the DCMI Element set can be inserted (title, creator, subject, description, publisher, contributor, date, type, format, identifier, source, language, relation, coverage, rights) as well as some refinement terms, such as the role of contributors, a spatial coverage - in WKT format geometries, or using GIS - and the type of relationship between one document and another.

The app does not need a network connection to perform object recognition. An internet connection is required only upon first opening or to update local data, in order to download remote data from the online database. The app uses a web service to request data stored on Cult.

\subsection{Future development}

Regarding AI training and testing, possible developments of the work include comparison between different ML models compatible with the developed apps.

Tests are still needed also on picture taking and image preprocessing. We need to understand and estimate to what extend including the surrounding context and other disturbing factors can affect the training results.

One of the possible outputs from this research or related works can be the construction of an open datasets to test and benchmark ML applications to architecture and architectural heritage. As stated above, not much attention seems to have risen around these topics in data science communities.

Future improvements of the app interface and functionalities could enhance the interaction with $3 \mathrm{D}$ digital models by implementing Augmented Reality (AR) functions. Moreover, 
research can explore the possible benefits of connecting these visualization techniques and ML algorithms. As an example, a network connected app could lean on ML to download a specific — possibly disk space consuming — 3D object tracking model, in order to enable AR on large sets of targets.

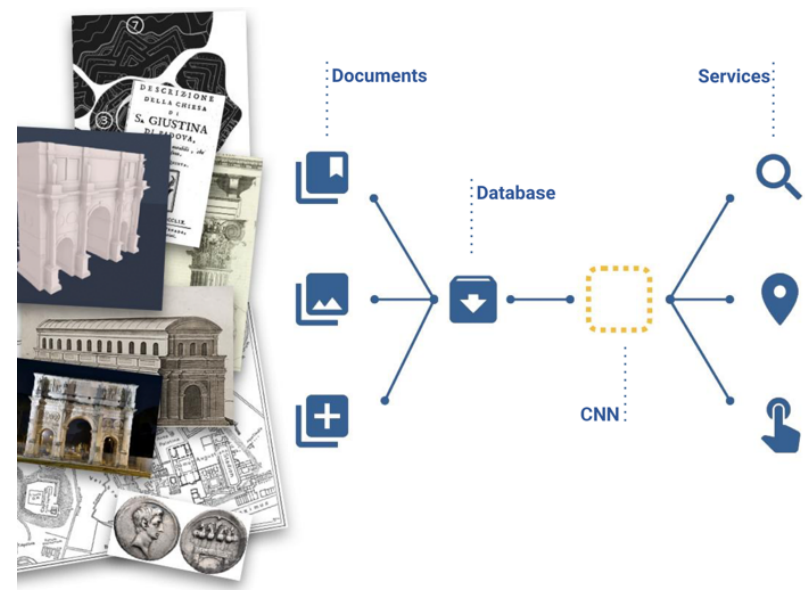

Figure 5. Schema of the connection between the Cult database and the functions available through the app.

\section{CONCLUSIONS}

This paper introduces the work of a multidisciplinary team to exploit AI technologies for image recognition in the architectural heritage field.

A role is proposed for automatic image recognition in localization techniques for sites of architectural interest.

The technology available on today's mobile devices offers the opportunity to rethink the way we access information. We propose a shift from user geo-localization to the localization of what a user is looking at, both in the physical space and in the complex topology of its related data.

Further developments of the integration of the proposed AI technologies and semantic spatial databases could:

1. make the system more scalable, storing online large amounts of data that can be retrieved when needed;

2. exploit the interoperability of the spatial information, i.e. connecting BIM data to the environment explored through the app;

3. allow access to a detailed information, i.e. reproducing the ML application at the scale of the single monument or building, thus recognizing its parts or categories of constructive elements, decorations, materials.

The Imperial Forum application is just one of the possible applications of the developed technology. As already stated by Amato et al. (2016) CNN models are general and can be trained to recognize a wide range of objects. This is why our team is planning to extend this project to other sites, covering different scales and time spans. However, we stress the importance of orienting similar experiences at optimizing $\mathrm{CNN}$ algorithms for architecture recognition.

This work could also point out possible connections between the virtual environment and the contemporary city. ML models could be trained to recognize building types or structural components, while the related information could integrate energy performance, structural behaviors, construction phases...

The underlying assumption of this research is that architecture has a key role in approaching technologically advanced tools.
Form is a means to identify physical, observable, tangible facts and it can be used to produce shared models of the complex and multi-layered urban space.

On this basis, our project intends to be a contribution to the recognition, structuring and operational use of architectural form.

\section{ACKNOWLEDGMENTS}

The team working on the project at the Politecnico di Torino is composed by Matteo Robiglio (full professor in Architectural and Urban Design), Francesca Frassoldati (associate professor in Architectural and Urban Design), Claudio Casetti (associate professor in Industrial and Information Engineering) and the author. The team is part of the Interdepartmental Center FULL_Future Urban Legacy Lab. The author is working on the SHAZARCH project as a $\mathrm{PhD}$ candidate in Architecture. History and Project at the department of Architecture and Design of the Politecnico di Torino. Louis N. Andrianaivo from the Department of Mathematics and Désirée Adiutori from the Department of Computational Sciences of the Università di Roma Tre trained the ML model and developed the Android prototype apps. The ReLOAD Research Lab of ArchitectURban Design of the Università di Padova has made available and maintained the Cult web service. The SHAZARCH project was initiated from an idea of Roberto D'Autilia and Paolo Desideri.

\section{REFERENCES}

Amato, G., Falchi, F., Gennaro, C., 2015. Fast Image Classification for Monument Recognition. Journal on Computing and Cultural Heritage 8, 1-25. https://doi.org/10.1145/2724727

Amato, G., Falchi, F., Vadicamo, L., 2016. Visual Recognition of Ancient Inscriptions Using Convolutional Neural Network and Fisher Vector. Journal on Computing and Cultural Heritage 9, 124. https://doi.org/10.1145/2964911

Apollonio, F.I., Gaiani, M., Benedetti, B., 2012. 3D reality-based artefact models for the management of archaeological sites using 3D GIS: a framework starting from the case study of the Pompeii Archaeological area. Journal of Archaeological Science, 39, pp. 1271-1287. https://doi.org/10.1016/j.jas.2011.12.034

Apple Inc., 2018. Xcode, Version 10.1, Apple Inc., https://www.archesproject.org (1 December 2018)

Google Inc., JetBrains s.r.o., 2018. Version 3.2, Google Inc., JetBrains s.r.o., https://developer.android.com/studio (1 December 2018)

Arches project, 2018. https://www.archesproject.org (1 December 2018).

Auer, M., Agugiaro, G., Billen, N., Loos, L., Zipf, A., 2014. Web-based Visualization and Query of semantically segmented multiresolution 3D Models in the Field of Cultural Heritage. ISPRS Annals of Photogrammetry, Remote Sensing and Spatial Information Sciences, II-5, pp. 33-39.

https://doi.org/10.5194/isprsannals-II-5-33-2014

Berners-Lee, T., Hendler, J., Lassila, O., 2001. The Semantic Web. Scientific American, 284, pp. 34-43.

Bortot, A., Boscaro, C., Cecchini, C., Francesca, C., Cundari, M.R., Palma, V., Panarotto, F., Siviero, L., 2017. TU-CULT. Rivelazioni architettoniche nelle chiese di Santa Giustina e di Santa Maria dei Servi a Padova. In: Territori e frontiere della rappresentazione/Territories and frontiers of representation. $39^{\circ}$ Convegno internazionale dei docenti e delle discipline della rappresentazione. Gangemi Editore, pp. 911-920.

Cerutti, E., Noardo, F., Spanò, A., 2015. Architectural Heritage Semantic Data Managing and Sharing in GIS. In: Proceedings of the 1st International Conference on Geographical Information 
Systems Theory, Applications and Management. SCITEPRESS Science and Technology Publications, Barcelona, pp. 121-128. https://doi.org/10.5220/0005387801210128

Chen, T., Wu, K., Yap, K.-H., Li, Z., Tsai, F.S., 2009. A Survey on Mobile Landmark Recognition for Information Retrieval. In: $M D M$ '09. IEEE Computer Society, pp. 625-630.

Chollet, F., Google Inc., Microsoft Inc., Keras contributors, 2018. Keras software library, Version 2.2.4, https://github.com/keras-team/keras (1 December 2018).

Cordts, M., Omran, M., Ramos, S., Rehfeld, T., Enzweiler, M., Benenson, R., Franke, U., Roth, S., Schiele, B., 2016. The Cityscapes Dataset for Semantic Urban Scene Understanding. In: 2016 IEEE Conference on Computer Vision and Pattern Recognition (CVPR), pp. 3213-3223. https://doi.org/10.1109/CVPR.2016.350

Cult project, 2018. http://cult.dicea.unipd.it (1 December 2018).

DCMI, 2018. http://dublincore.org/ (1 December 2018).

Doerr, M., 2003. The CIDOC Conceptual Reference Module: An Ontological Approach to Semantic Interoperability of Metadata. AI magazine, 24, pp. 75-92.

Eide, O., Felicetti, A., Ore C., E., D'Andrea, A., Holmen, J., 2008. Encoding Cultural Heritage Information for the Semantic WEB. Procedures for Data Integration through CIDOC-CRM Mapping. In proceedings: EPOCH Conference on Open Digital Cultural Heritage Systems, ARCHAEOLINGUA, Budapest.

Gada, S., Mehta, V., Kanchan, K., Jain, C., Raut, P., 2017. Monument Recognition Using Deep Neural Networks. In: 2017 IEEE International Conference on Computational Intelligence and Computing Research (ICCIC), pp. 1-6. https://doi.org/10.1109/ICCIC.2017.8524224

Goodfellow, I., Bengio, Y., Courville, A., 2016. Deep Learning. The MIT Press, Cambridge.

Google Inc., Tang, Y., 2018. TensorFlow software library, Version 1.12, https://github.com/tensorflow/tensorflow (1 December 2018)

Hosny, A., Parmar, C., Quackenbush, J., Schwartz, L.H., Aerts, H.J.W.L., 2018. Artificial intelligence in radiology. Nature Reviews Cancer, 18, pp. 500-510. https://doi.org/10.1038/s41568-018-0016-5

Hor, A.-H., Jadidi, A., Sohn, G., 2016. BIM-GIS Integrated Geospatial Information Model Using Semantic Web and RDF Graphs. ISPRS Annals of Photogrammetry, Remote Sensing and Spatial Information Sciences, III-4, pp. 73-79.

https://doi.org/10.5194/isprsannals-III-4-73-2016

Howard, A.G., Zhu, M., Chen, B., Kalenichenko, D., Wang, W. Weyand, T., Andreetto, M., Adam, H., 2017. MobileNets: Efficient Convolutional Neural Networks for Mobile Vision Applications. arXiv:1704.04861 [cs].

INCEPTION project, 2018. http://inception-project.eu (1 December 2018).

Kaggle Inc., 2018. https://www.kaggle.com (1 December 2018).

Kakali, C., Lourdi, I., Stasinopoulou, T., Bountouri, L., Papatheodorou, C., Doerr, M., and Gergatsoulis, M., 2007. Integrating Dublin Core metadata for cultural heritage collections using ontologies. In proceedings: International Conference on Dublin Core and Metadata Applications, Sept. 1-4. e-LIS, São Paulo.

Liu, X., Wang, X., Wright, G., Cheng, J., Li, X., Liu, R., 2017. A State-of-the-Art Review on the Integration of Building Information Modeling (BIM) and Geographic Information
System (GIS). ISPRS International Journal of Geo-Information, 6, 53. https://doi.org/10.3390/ijgi6020053

Maietti, F., Di Giulio, R., Piaia, E., Medici, M., Ferrari, F., 2018. Enhancing Heritage fruition through 3D semantic modelling and digital tools: the INCEPTION project. IOP Conference Series: Materials Science and Engineering, 364.

https://doi.org/10.1088/1757-899X/364/1/012089

Murphy, M., McGovern, E., Pavia, S. (2009). Historic building information modelling (HBIM). Structural Survey, 27(4), pp. 311-327.

Myers, D., Dalgity, A., Avramides, I., 2016. The Arches heritage inventory and management system: a platform for the heritage field. Journal of Cultural Heritage Management and Sustainable Development, 6, pp. 213-224. https://doi.org/10.1108/JCHMSD02-2016-0010

Oldman, D., de Doerr, M., de Jong, G., Norton, B., Wikman, T., 2014. Realizing Lessons of the Last 20 Years: A Manifesto for Data Provisioning and Aggregation Services for the Digital Humanities (A Position Paper). D-Lib Magazine, 20 https://doi.org/10.1045/july2014-oldman

Saygi, G., Agugiaro, G., Hamamcioğlu-Turan, M., Remondino, F., 2013. Evaluation of GIS And BIM Roles for the Information Management of Historical Buildings. ISPRS Annals of Photogrammetry, Remote Sensing and Spatial Information Sciences, II-5/W1, pp. 283-288.

https://doi.org/10.5194/isprsannals-II-5-W1-283-2013

Saygi, G., Remondino, F., 2013. Management of Architectural Heritage Information in BIM and GIS: State-of-the-Art and Future Perspectives. International Journal of Heritage in the Digital Era, 2, pp. 695-713.

https://doi.org/10.1260/2047-4970.2.4.695

Scianna, A., Gristina, S., and Sciortino, R. (2013). Integrazione di sistemi GIS FOSS e modelli dati 3D PDF per la fruizione multimediale di beni monumentali e archeologici: il Castello di Maredolce a Palermo. In: Stanco, F., and Gallo G. (eds). Free, libre and open source software e open format nei processi di ricerca archeologica. Eight edn. Archaeopress.

Soler, F., Melero, F.J., Luzón, M.V., 2017. A complete 3D information system for cultural heritage documentation. Journal of Cultural Heritage, 23, pp. 49-57.

https://doi.org/10.1016/j.culher.2016.09.008

Song, Y., Wang, X., Tan, Y., Wu, P., Sutrisna, M., Cheng, J., Hampson, K., 2017. Trends and Opportunities of BIM-GIS Integration in the Architecture, Engineering and Construction Industry: A Review from a Spatio-Temporal Statistical Perspective. ISPRS International Journal of Geo-Information, 6, 397. https://doi.org/10.3390/ijgi6120397

Volk, R., Stengel, J., Schultmann, F., 2014. Building Information Modeling (BIM) for existing buildings - Literature review and future needs. Automation in Construction, 38, pp. 109-127. https://doi.org/10.1016/j.autcon.2013.10.023

Webb, S., 2018. Deep learning for biology. Nature, 554, pp. 555557. https://doi.org/10.1038/d41586-018-02174-z 